\title{
BMJ Open Is the introduction of violence and injury observatories associated with a reduction of violence in adult populations? Rationale and protocol for a systematic review
}

\author{
Ardil Jabar, ${ }^{1}$ Dylan Barth, ${ }^{2}$ Richard Matzopoulos, ${ }^{1}$ Mark Emmanuel Engel ${ }^{2}$
}

To cite: Jabar A, Barth D, Matzopoulos R, et al. Is the introduction of violence and injury observatories associated with a reduction of violence in adult populations? Rationale and protocol for a systematic review. BMJ Open 2015;5: e007073. doi:10.1136/ bmjopen-2014-007073

- Prepublication history for this paper is available online. To view these files please visit the journal online (http://dx.doi.org/10.1136/ bmjopen-2014-007073).

Received 31 October 2014 Revised 23 April 2015 Accepted 27 April 2015

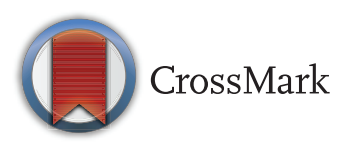

${ }^{1}$ Faculty of Health Sciences, School of Public Health and Family Medicine, University of Cape Town, Cape Town, Western Cape, South Africa ${ }^{2}$ Faculty of Health Sciences, Department of Medicine, University of Cape Town, Cape Town, Western Cape, South Africa

Correspondence to Dr Ardil Jabar; A.jabaroo@gmail.com

\begin{abstract}
Introduction: The violence and injury observatories developed in Colombia and found throughout the Americas and Western Europe aim to maximise interinstitutional cooperation, information-sharing, analysis and security policy development initiatives to enhance governance. The purpose of the violence and injury observatories is directed towards preventing crime and violence at the local and regional levels. To date, there has been no systematic review of the literature to present a succinct review of the evidence. We therefore sought to summarise the evidence from existing studies on the contribution of violence and injury observatories towards violence prevention.
\end{abstract}

Methods and analysis: A number of databases will be searched, supplemented by the same keyword searches in the grey literature. Search terms will include studies published from 1 January 1990 to 30 October 2014. Study quality will be assessed using a validated quality assessment tool. Two researchers will independently assess articles for study eligibility to reduce bias, minimise errors and enhance the reliability of findings. Disagreements will be resolved by consensus among three authors. This review protocol has been published in the PROSPERO International Prospective Register of systematic reviews, registration number 2014:CRD42014009818.

Dissemination: There is a paucity of evidence for the effectiveness of violence and injury observatories and their influence on violence in an adult population. We plan to address this gap in knowledge by way of a systematic review and meta-analysis outlined in this abstract. We anticipate that the results could be used by researchers and policymakers to help inform them of the efficacy of violence and injury observatories and their broader role in contributing to violence prevention.

Trial registration number: CRD42014009818.

\section{INTRODUCTION}

Violence is now recognised officially as a global health issue, with the WHO reporting

\section{Strengths and limitations of this study}

- To the best of our knowledge, this is the first systematic review protocol that will attempt to assess the impact of violence and injury observatories in contributing to violence prevention among an adult population.

- This study will inform research practice and future studies on the effectiveness of observatories.

- Inferences regarding outcomes cannot be drawn from the present protocol, but will be reported following the systematic review and metaanalysis described herein.

- While causality cannot be inferred from this review, only an association can be inferred.

that 1.6 million people die annually from violence. ${ }^{1}$ South Africa's injury burden is very high, particularly for homicide, which is seven times the global average. ${ }^{2}$ The 2010 Global burden of disease study, which bases its estimates on vital registration, found interpersonal violence to be the third leading cause of premature death in South Africa, accounting for approximately $4.4 \%$ of all years of life lost. ${ }^{3}$ Mortuary data record significantly more homicides, and thus the contribution of interpersonal violence to the disease burden is likely to be considerably higher. ${ }^{4}$

The public health approach to injury prevention includes three elements, namely risk assessment, interventions development and programme evaluation. ${ }^{5}$ Important to facilitating this approach, is a surveillance system capable of providing essential information for the assessment phase in order to develop appropriate interventions and programme evaluation methods. ${ }^{67}$

Ongoing surveillance can monitor the incidence of injury, identify risk factors and contribute to the planning and evaluation of 
injury prevention programmes. ${ }^{8} 9$ Injury surveillance is widely recognised as a critical prerequisite for effective injury prevention. ${ }^{10}$ Injury surveillance utilises a variety of data sources, from mortality and hospital discharge data to emergency department registry data, surveys and police, fire or ambulance records. ${ }^{6}$

Several studies in different countries have highlighted injury surveillance systems' ability to identify contextual risk factors and the larger contribution to injury prevention programmes. ${ }^{9-14}$ Furthermore, substantial success in notable reductions in a number of violence outcomes has been reported in observational studies and shown in trend analyses from centres in Colombia and throughout South America. ${ }^{15}{ }^{16}$ Directed towards preventing crime and violence at the local and regional levels, these violence and injury observatories aim to maximise interinstitutional cooperation, information-sharing, analysis and security policy development initiatives to enhance governance. $^{7}$

An observatory is primarily a tool to help in decisionmaking based on the principle that a better knowledge of situations affecting security will make it possible to ensure more targeted and thus more effective interventions. Although a diagnostic tool measuring the degree of violence in a defined region, over time an observatory also makes it possible to monitor and evaluate the impact of measures adopted.

To date, there has been no systematic review of the literature to present a succinct review of the evidence. We therefore sought to summarise the evidence from existing studies on the contribution of violence and injury observatories towards violence prevention.

\section{METHODS}

This review protocol has been published in the PROSPERO International Prospective Register of systematic reviews (http://www.crd.york.ac.uk/PROSPERO), registration number 2014: CRD42014009818.

\section{CRITERIA FOR CONSIDERING STUDIES FOR THIS REVIEW Type of studies}

We will include randomised controlled trials (RCTs), non-randomised controlled trials, quasi-experimental designs, prospective and retrospective cohort studies and controlled before-and-after CBA studies. In the absence of these, cross-sectional studies will be included. Studies performed in general or specific populations and in hospitals or clinics will be included. Additionally, studies performed in any country and published in any language will be included.

\section{Types of participants}

Participants for this study will include adults $\geq 18$ years of age who are located within the catchment areas of the observatory study sites.

\section{Types of interventions/exposures}

For purposes of the systematic review, we will use the term 'observatory' to denote a surveillance system that collects data from multiple sources, for example, crime, clinical and forensic data, whereas injury surveillance systems almost exclusively focus on the use of injury data alone. We will include observatories/injury surveillance systems that address violence prevention and whether these reduce violence in adult populations. All surveillance systems that focus specifically on the collection of violence and injury data will be included in this review.

\section{Types of outcome measures}

Violence will be defined as the intentional threat or use of physical force against oneself, another person or a group or community that results in injury, death, psychological harm, maldevelopment or deprivation. ${ }^{17}$ The outcome measures will be based on the Organisation of American States (OAS) regional system of standardised indicators in peaceful coexistence and citizen security, ${ }^{8}$ as they represent the largest member organisation of crime and violence observatories worldwide, and will include measures obtained by administrative record or surveys.

\section{Primary outcomes}

Primary outcomes will include murder/homicide, suicide, transit death, unintentional injury death, sexual violence and intrafamily/family/domestic violence. ${ }^{8}$

\section{Secondary outcomes}

Secondary outcomes will include aggravated assault, crime victimisation and the perception of insecurity, fear or risk. ${ }^{8}$

\section{SEARCH METHODS FOR IDENTIFICATION OF STUDIES}

The search of databases and the grey literature will be performed by AJ with the help of the University of Cape Town librarian, to identify all relevant studies available by 30 October 2014, regardless of language or publication status. Peer-reviewed journal articles and the grey literature (unpublished, internal or non-reviewed papers and reports) will also be searched.

\section{Database}

We will search the following electronic databases: Pubmed, Sociological abstracts and International bibliography of the social sciences (IBSS) and Education sources information centre (ERIC) via Proquest, PsycINFO and Cumulative index to nursing and allied health literature (CINAHL) and Humanities International via Ebscohost, SCOPUS, Cochrane Collaboration, Campbell Collaboration, Social Care Online, National Criminal Justice Reference Service, Web of Knowledge and Regional databases of the WHO. Furthermore, the following websites will be searched for relevant literature: websites of the WHO Violence Prevention Alliance (http://www.who.int/violenceprevention/en/), 
Blueprints for Violence Prevention (http://www.colorado. edu/cspv/blueprints), the Community Guide (http://www. thecommunityguide.org/violence/index.html), Centers for Disease Control and Prevention (http://www.cdc.gov/ ViolencePrevention/index.html), The World Bank (http://www.worldbank.org), the Juarez violence and injury observatory (http://observatoriodejuarez.org/ dnn/ENGLISH.aspx) and the Medical Research Council (MRC) burden of disease research unit (http://www. mrc.ac.za/bod/bod.htm).

In addition, the following conference proceedings will be searched for relevant abstracts: International Conference on Crime Observatories, United Nations Congress on Crime Prevention and Criminal Justice, Global Violence Reduction conference, Annual meeting of Violence Prevention Alliance and the International society for violence and injury prevention international conference. We will use both text words and medical subject headings terms. The terms will be used in varying combinations. The specific database will determine the literature search strategy employed as shown in table 1. Reviewers will also search reference lists of the relevant studies identified.

\section{DATA COLLECTION AND ANALYSIS}

The Cochrane Handbook of Systematic Reviews for Interventions ${ }^{9}$ will serve as the reference for methods employed in this study.

\section{Selection of studies for inclusion}

Review authors will use a screening guide developed by AJ to ensure that inclusion criteria are consistently applied. Two review authors (AJ and DB), working independently, will screen the titles and abstracts of all studies identified through the literature searches for eligibility. Full texts of potentially eligible studies will be obtained by AJ. The two authors (AJ and DB) will independently assess the full text of each article for eligibility, and compare their results. Reviewer agreement will be reported by a $\kappa$ statistic. Discrepancies will be resolved through discussion and consensus, consulting a third author (MEE) to resolve any persistent disagreements. Reviewers will document the reasons for all studies excluded from the systematic review.

\section{Assessment of risk of bias in included studies}

Two reviewers will assess all included studies using the Effective Public Health Practice Project (EPHPP) questionnaire, which is a quantitative study assessment tool to identify methodological issues. ${ }^{10}$ The criteria used to assess the risk of bias in RCTs will be random sequence generation; allocation concealment; blinding of participants and study personnel; blinding of outcome assessors; incomplete outcome data; selective outcome reporting; other sources of bias and overall risk of bias, in accordance with the methods used by the Cochrane Collaboration and the EPHPP tool. ${ }^{9}$ The criteria used for risk of bias assessment for non-randomised studies will include selection bias (dealing with confounding, adjustment and comparability of groups); performance bias (in terms of the fidelity of the interventions); detection bias (regarding unbiased and correct assessment of outcomes, including blinding of assessors); attrition bias (with regard to completeness of sample, follow-up and data); and reporting bias (with regard to publication biases and selective reporting of results). ${ }^{9}$ Studies will be scored as having low, high or unclear risk of bias. Any disagreements between the two authors in the assessment of risk of bias will be resolved in discussion and consensus and the consultation of a third author when necessary.

\section{Data extraction and management}

Two authors will independently extract descriptive and outcome data for each included article using a standardised data collection form, resolving any discrepancies by discussion and consensus, failing which a third author (MEE) will arbitrate. The final data will be entered into the Cochrane Collaboration Review Manager V.5.1 statistical software (http://ims.cochrane. org/RevMan) by AJ while DB will cross-check the data

\begin{tabular}{|c|c|}
\hline Search & PubMed \\
\hline \#1 & (violence and injury observator*) OR (injury surveillance system) OR (crime observator*) \\
\hline \#2 & $\begin{array}{l}\text { ("domestic abuse" OR "physical abuse" OR "partner abuse*" OR violent OR violence OR assault OR homicide OR } \\
\text { gang OR gangs OR gang violence OR bully OR aggression OR aggressive OR robbery OR assault OR GBH OR } \\
\text { contact crime OR interpersonal violence OR murder OR homicide OR aggravated assault OR robbery OR suicide } \\
\text { OR transit death OR non intentional death OR kidnappings OR theft OR robberies OR rape OR sexual crimes OR } \\
\text { assault OR physical violence OR aggression OR sexual violence OR violent crime OR violent crime conviction) }\end{array}$ \\
\hline \#3 & $\begin{array}{l}\text { (prevention OR preventing OR prevent OR reduction OR reduce OR decrease OR decreased OR decreasing OR } \\
\text { decline OR declining OR control OR controlling OR impact OR effect OR effects OR affect OR affecting OR affects } \\
\text { OR change OR changing OR changes OR intervene OR intervention) }\end{array}$ \\
\hline \#4 & adult \\
\hline \#5 & \#1 AND \#2 \\
\hline \#6 & \#3 AND \#4 \\
\hline
\end{tabular}


entered to ensure that there are no data entry errors. References will be managed using Refworks V.2.0. ${ }^{11}$

\section{Data synthesis including assessment of heterogeneity}

Data analysis will be managed using the Cochrane Collaboration Review Manager V.5.1 statistical software (http://ims.cochrane.org/RevMan) with the outcomes of interest being either dichotomous or continuous. Risk ratios and their respective 95\% CI and $\mathrm{p}$ values will be calculated for dichotomous outcomes, and mean differences and SDs will be calculated for continuous outcomes. Standardised mean differences will be calculated where outcomes are measured using different scales. ${ }^{12}$ Heterogeneity will be assessed by examining types of participants, interventions and outcomes in each study with the intention to pool data and estimate effect sizes using a fixed-effects model only from studies in which outcomes are judged to be homogeneous. Alternately, the random-effects model will be employed. Statistical heterogeneity in each meta-analysis will be assessed using the $\chi^{2}$ test and quantified using the $\mathrm{I}^{2}$ statistic. ${ }^{13}$ The findings will be discussed as a narrative summary if the heterogeneity remains significant. Included studies will be summarised in tables to highlight the main existing evidence.

\section{Subgroup analyses}

Subgroup analyses will be performed by intervention subtypes: high income areas versus low income areas and high concentration of violence areas versus low concentration of violence areas. Analysis will be further stratified by study design (randomised controlled studies separate from non-randomised studies) and intervention type (surveillance system collecting violence and injury data only vs a system that collects violence and injury data as well as crime data). We will also conduct a subgroup comparison of self-reported violence outcome behaviours versus verified criminal records as well as according to age categories and country setting.

\section{Assessment of quality of evidence}

We will use the grading of recommendations assessment, development and evaluation (GRADE) approach to assess the quality of evidence for the contribution of the observatory towards violence prevention. ${ }^{14}$ 'The GRADE approach specifies four levels of quality ranging from high to very low, with the highest quality rating denoting a confidence that the true effect lies close to the estimate of the effect. Quality will be rated according to an a priori identification of potential participant-centred outcomes, including benefits and harms. ${ }^{15}$

Two authors will independently assign the grade scores and compare results as per the process for the recording of previous aspects of the study. Discrepancies will be resolved by consensus discussion between the two primary reviewers (AJ and $\mathrm{DB}$ ), with arbitration by a third reviewer (MEE) as necessary'

\section{Sensitivity analyses}

Multiple sensitivity analyses will be conducted. We will first determine whether the study design (RCT vs nonrandomised study) could influence the results of the meta-analysis. Second, the model of the statistical method (random-effects model vs fixed-effects model) will be evaluated to determine if this could change the results. We will determine the impact of excluding studies with a high risk bias on the results, with emphasis on allocation concealment, blinded outcome assessment and losses to follow-up (with a cut-off of 25\% loss to follow-up). Further sensitivity analyses will be considered if necessary.

\section{REPORTING OF THIS REVIEW}

This review's findings will be reported in several ways. Preferred Reporting Items for Systematic Reviews and Meta-Analyses (PRISMA) flow diagrams will be used to summarise the study selection process. ${ }^{15}$ The $\kappa$ statistic ${ }^{16}$ will be used to assess agreements between the full-text screening, data extraction and risk of bias assessment by the two authors (AJ and $\mathrm{DB}$ ). Where necessary, we will adapt the reporting to ensure that all items relevant to this review are included.

\section{ETHICS AND DISSEMINATION}

Ethics is not required for this study as it utilises public health data. The findings of this study will be widely disseminated through peer-reviewed publications, conference presentations and submitted to relevant authorities in national departments of health. Updates of the review will be completed to inform and guide violence preventative measures.

\section{IMPLICATIONS}

While the criminal justice system remains the primary tool for addressing violence in South Africa, evidencebased interventions for prevention are increasingly employed, with public health assuming a more central role in policymaking. ${ }^{18}$ The findings of this systematic review may provide evidence for the introduction of injury surveillance systems for the management of violence in our context. While we acknowledge that causality cannot necessarily be inferred from conducting a systematic review, we can, however, quantify a level of effect of an association between the introduction of an observatory and a subsequent decrease in levels of violence. Furthermore, policy implications regarding the results of the review may be that there is a greater acceptance by stakeholders in the safety cluster that security policies must be based on reliable, objective information. Finally, the implications for future research may be to understand how observatories influence social change.

Acknowledgements The authors would like to acknowledge the critical input and support of the Evidence-Based Medicine Research Support Unit, Faculty of Health Sciences, University of Cape Town. 
Contributors AJ conceived of the review. AJ and DB wrote the first draft and all authors edited the subsequent versions of the draft. AJ and DB developed the protocol, will conduct the searches and extract the data. MEE and RM will oversee the final analysis of the data. All authors have reviewed and accepted the final version of the protocol and given their permission for publication.

Funding The PhD from which this study emanated was funded by the Medical Research Council of South Africa under the MRC Clinician Researcher Programme.

Competing interests None declared.

Provenance and peer review Not commissioned; externally peer reviewed.

Open Access This is an Open Access article distributed in accordance with the Creative Commons Attribution Non Commercial (CC BY-NC 4.0) license, which permits others to distribute, remix, adapt, build upon this work noncommercially, and license their derivative works on different terms, provided the original work is properly cited and the use is non-commercial. See: http:// creativecommons.org/licenses/by-nc/4.0/

\section{REFERENCES}

1. Krug EG, Dahlberg LL, Mercy JA, et al. World report on violence and health. Geneva: 2002. http://whqlibdoc.who.int/hq/2002/9241545615.pdf

2. Bradshaw D, Groenewald P, Laubscher R, et al. Initial burden of disease estimates for South Africa, 2000. 2003. http://www.ncbi.nlm. nih.gov/pubmed/14635557

3. Lozano R, Naghavi M, Foreman K, et al. The Global Burden of Disease Study 2010. Washington DC: 2010. http://www.healthdata. org/sites/default/files/files/country_profiles/GBD/ihme_gbd_country_ report south africa.pdf

4. Matzopoulos R, Prinsloo M, Bradshaw D, et al. The injury mortality survey: a national study of injury mortality levels and causes in South Africa in 2009. Cape Town: South African Medical Research Council, 2013
5. Nilsen P, Bourne M, Coggan C. Using local injury surveillance for community-based injury prevention: an analysis of Scandinavian WHO Safe Community and Canadian Safe Community Foundation programmes. Int J Inj Contr Saf Promot 2007;14: 35-43.

6. Lyons R, Jones S, Kemp A, et al. Development and use of a population based injury surveillance system: the All Wales Injury Surveillance System (AWISS). Inj Prev 2002;8:83-6.

7. Joanie P, Ferland S, Bruneau S. Crime observatories: International experience directory. Montreal: International Centre for the Prevention of Crime, 2009.

8. OAS. The regional system of standardized indicators in peaceful coexistence and citizen security project. 2014. http://www. seguridadyregion.com/ (accessed 29 Nov 2014).

9. Higgins J, Green S, eds. Cochrane handbook for systematic reviews of interventions. Version 5.0.2 [updated September 2009]. The Cochrane Collaboration, 2009:190-202. www.cochrane-handbook.org

10. Armijo-Olivo S, Stiles $\mathrm{C}$, Hagen $\mathrm{N}$, et al. Assessment of study quality for systematic reviews: a comparison of the Cochrane Collaboration Risk of Bias Tool and the Effective Public Health Practice Project Quality Assessment tool: methodological research. J Eval Clin Pract 2012;18:12-18.

11. Refworks. 2009. https://www.refworks.com/

12. Borenstein M. Introduction to meta-analysis. Chichester, UK: John Wiley \& Sons, 2009.

13. Higgins J, Thompson S. Quantifying heterogeneity in a meta-analysis. Stat Med 2002;21:1539-58.

14. Balshem $\mathrm{H}$, Helfand $\mathrm{M}$, Schunemann $\mathrm{H}$, et al. GRADE guidelines: 3 Rating the quality of evidence. J Clin Epidemiol 2011;64: 401-6.

15. Moher D, Liberat A, Tetzlaff J, et al. Preferred reporting items for systematic reviews and meta-analyses: the PRISMA Statement. J Clin Epidemiol 2009;62:1006-12.

16. Landis J, Koch $\mathrm{G}$. The measurement of observer agreement for categorical data. Biometrics 1977;33:159-74.

17. Sethi D. The role of public health in injury prevention. Rome: 2007 http://www.euro.who.int/ data/assets/pdf file/0010/98803/Policy briefing_1.pdf 